\title{
RADIATION PROTECTIVE STRUCTURES ON THE BASE OF A CASE STUDY FOR A MANNED MARS MISSION
}

\author{
Andreas Borggräfe \\ Space Research Group, RWTH Aachen University, Germany \\ Andreas.Borggraefe@rwth-aachen.de \\ Michael Quatmann \\ Department of Aerospace and Lightweight Structures \\ RWTH Aachen University, Germany \\ quatmann@ilb.rwth-aachen.de \\ Daniel Nölke \\ Department of Aerospace and Lightweight Structures \\ RWTH Aachen University, Germany \\ noelke@ilb.rwth-aachen.de
}

\begin{abstract}
Plans for interplanetary manned space missions imply significant risks arising from human's exposure to the hostile space environment. Thus the design of reliable protection systems against the ionising cosmic radiation becomes one of the most relevant issues. In this paper the composition and magnitude of the atmospheric radiation on the planetary surface and for typical interplanetary transfer configurations have been analyzed. The investigation based on prior NASA and ESA mission results, using a manned mission to planet mars as a case study. According to this, the time-dependent character of the consistency of cosmic radiation has been taken into account, which is justified by the interdependence of the radiation magnitude to the solar cycle. With regard to this paper it implies even solar particle events. The results have been compared to the protective character of different materials potentially usable as a habitat's structural shell and for interplanetary spacecrafts. The investigation aimed on particle energy degradation rates and reduction of secondary particle production. In this regard the physical process of absorbing effectiveness against particle radiation has been examined by analytical calculation and given scientific results, depending on thickness and molecular composition of the materials. The most suitable materials have been used for shield design proposals using different configurations, evaluating the use of aluminium, water tanks and polyethylene bricks.
\end{abstract}

\section{Introduction}

The vision of human interplanetary space exploration primarily depends on protection of the astronauts from the hazadrous radiational environment present outside the earth's magnetic field. The effects of space radiation on the human body can be a mission limiting factor and thus must be taken into account during design phases for interplanetary manned missions with special emphasis.

Within this study, a first conceptual approach has been perfomed to examine the potential of alter- native materials for passive radiation protection of astronauts within an interplanetary spacecraft to planet Mars. Therefore, reference Mars missions scenarios have been adopted as a conceptual baseline [1] . The calculations are done utilizing two currently available radiation transport codes: HZETRN2005 [2] from NASA/LaRC and Geant4/Mulassis [3] from CERN/ESA. The codes are based on essentially different numerical approaches, deterministic and Monte Carlo method. Energy spectra of radiation fields present in space during the solar cycle have been used as input for 
the codes. Any numerical approach is fairly dependent on how these codes represent the real radiation environment in space, the propagation of particles through the shield matter and the (human) target in terms of body tissue. The computational modeling of the human body is an essential basis for radiation risk assessment, because the spatial distribution of irradiance within the body determines the overall dose received and resulting health risks. This modeling turned out to provide the most outstanding problem within the calculations and set a procedural boundary in this study. On the other hand, the results for the calculated set of materials give a reliable qualitative performance comparison and are further analyzed in terms of applicability for a material-conditioned shield design. To further qualitatively investigate the total dose received by the crew during their Mars mission, reference results from currently available radiation studies have been compared to the chosen mission profiles to determine the preferable mission strategy.

\section{Radiation Environment in Space}

The composition of the radiation fields outside the magnetic field of earth are spatially and temporarily variable and are commonly divided into four different sources and types [4]:

Galactic Cosmic Radiation (GCR): fully ionized particles from outside the solar system, 91\% protons, $8 \% \alpha$-particles, $1 \%$ heavy nuclei

Solar Particle Events (SPE): temporal and sudden ejection of particles from the sun's heliosphere, mainly protons, high flux density

Trapped Radiation Fields (van Allen Belts): trapped cosmic radiation particles along the field lines of the terrestrial magnetic field, mainly protons and electrons

\section{Secondary Radiation on Planets: spectrum} interaction with planetary atmosphere or surface, mainly neutrons

The GCR consists of nuclei of almost all known chemical elements and energies in the range of several tens up to $10^{12} \mathrm{MeV}$ per nucleon. Their distribution is assumed almost isotropic throughout open space [4]. Even though the number of high charge and energetic (HZE) particles is relatively small, their contribution to the deployed dose is significant due to their highly ionizing character and thus hazadrous biological effects. The intensity of the GCR varies in dependance to the sun's eleven year cycle (figure 1) and reaches a minimum during solar maximum conditions with fluxes approximately half as large as during solar minimum.

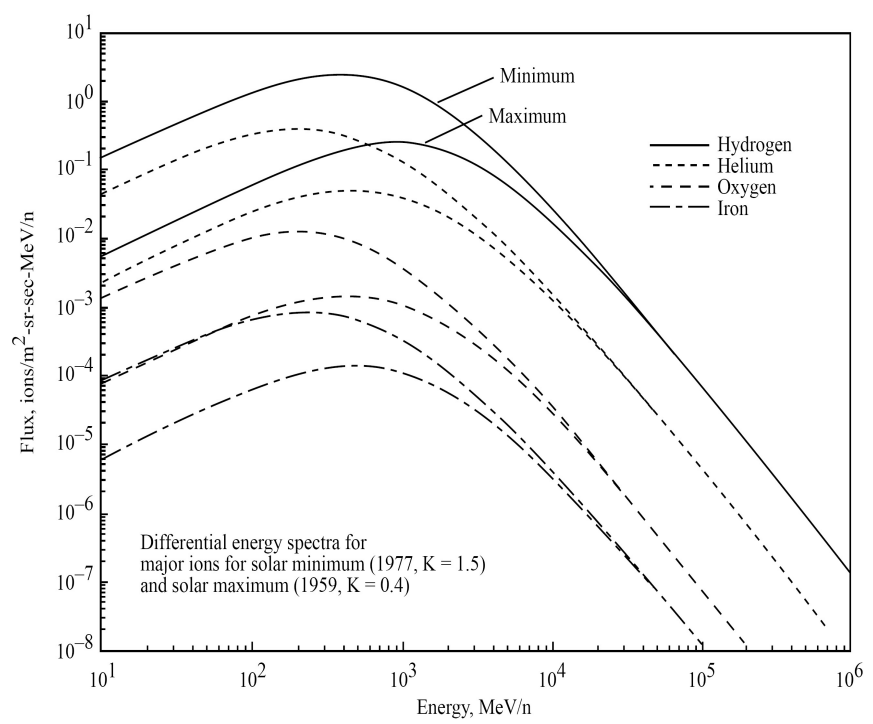

Figure 1: Differential GCR spectra during solar minimum and maximum [5]

Solar particle events (SPE) are widely accepted to be caused by coronal mass ejections, accelerating the particles to smaller kinetic energies in comparison to the GCR, but much higher particle fluences. The enormous flux ratios are the reason why SPE can deliver a very high dose in a short period of time since SPE's typically last from several hours to few days. These events are stochastic in nature and are not yet predictable with sufficient warning time. The most intense SPE's in terms of proton flux and energies are depicted in figure 2.

In contrast to free space conditions, the radiation environment in planetary orbits and on surface will be reduced up to $40 \%$ due to shielding of the planet's mass, since solar and galactic radiation is essentially isotropic. 


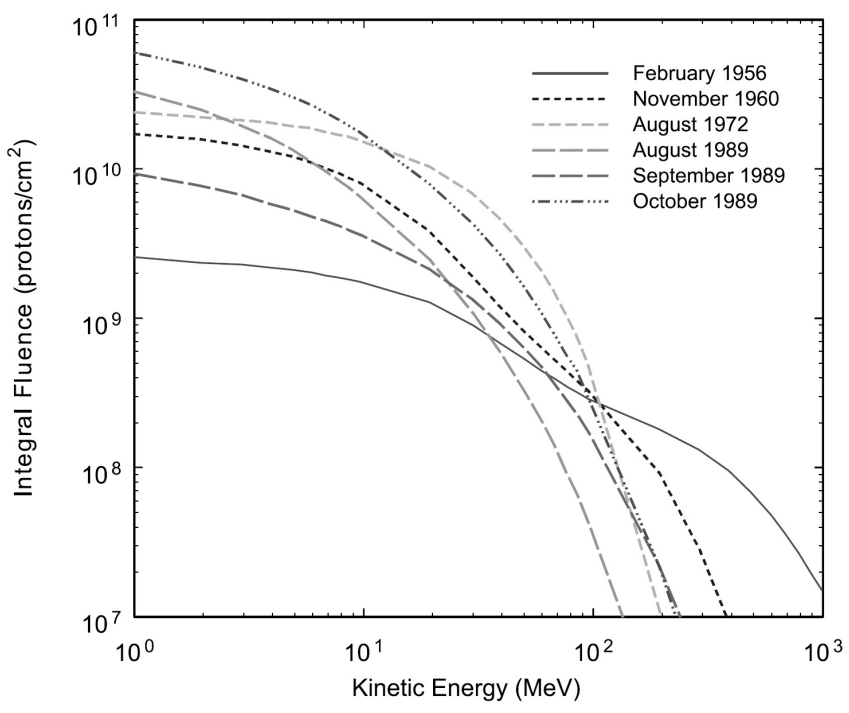

Figure 2: Integral proton fluences for several major SPEs over the last four solar cycles 6 ]

\begin{tabular}{ccc}
\hline $\begin{array}{c}\text { Altitude } \\
(\mathrm{km})\end{array}$ & $\begin{array}{c}\text { Low-density model } \\
\left(\mathrm{g} \mathrm{CO} / \mathrm{cm}^{2}\right)\end{array}$ & $\begin{array}{c}\text { High-density model } \\
\left(\mathrm{g} \mathrm{CO} / \mathrm{Cm}^{2}\right)\end{array}$ \\
\hline 0 & 16 & 22 \\
4 & 11 & 16 \\
8 & 7 & 11 \\
12 & 5 & 8 \\
\hline
\end{tabular}

Table 1: Areal density of martian $\mathrm{CO}_{2}$ atmosphere depending on altitude [5]

Although Mars has no significant magnetic field, the thin $\mathrm{CO}_{2}$ atmosphere (about $2 \%$ of thickness of earth's atmosphere) will provide additional shielding for the astronauts while staying on the martian surface. In contrast, earth's atmosphere provides shielding comparable to an approx. $10 \mathrm{~m}$ thick water column. Table 1 shows the areal density of the $\mathrm{CO}_{2}$ atmosphere for high and low density model [5].

\section{Effects of Ionizing Radiation}

High energetic ionizing radiation poses a significant threat for astronauts during missions in LEO and more severe on interplanetary missions in free space, where planetary magnetic shields and the shadowing effects of planetary masses have no effect.

\subsection{Passage of Particles Through Matter}

The nuclear particle species in cosmic radiation may generally be classified in two different kinds: directly ionizing (protons, $\alpha$ - and $\beta$-particles, higher $\mathrm{Z}$ nuclei) and indirectly ionizing particles (neutrons, photons). Directly ionizing, charged particles primarily lose their energies in discrete atomic excitations and ionizations of the target nuclei's electrons. Indirect ionizing, uncharged particles submit their energy through elastic and inelastic nuclear scattering in case of neutrons and Photo/Comptoneffect and pair production in case of photons (X-rays, $\gamma$-rays).

The direct ionization process is described by the particle's linear energy transfer (LET), which can be approximately quantified by the Bethe Bloch formula [7]. In general, the LET $\left(\frac{d E}{d x}\right)$ describes the average energy $\mathrm{dE}$ locally imparted to the medium by a charged particle of specific energy in traversing a distance $\mathrm{dx}$. The unit of the LET is mostly given as $\mathrm{keV} / \mu \mathrm{m}$. The traversing particle interacts with the electron hull of the material's atoms through its magnetic field, whereas it experiences elastic scattering at the electron cloud. $\frac{d E}{d x}$ is directly dependent on the particle's energy, charge, mass and on the density of atoms in the absorbing material or tissue.

The shown relations so far only describe the electronic energy loss of the cosmic particles and do not include nuclear energy losses due to nuclear scattering and nuclear fragmentation processes when the ion hits a nucleus. As addressed above, indirectly ionizing particles like neutrons also interact in this way. Three types are relevant: During elastic scattering the projectile and target nucleus are left intact and only their momenta may be changed. In contrast, inelastic scattering causes the projectile to lose a certain amount of energy and excites the target nucleus. Most important, through nuclear fragmentation (deep inelastic scattering) the target will be destroyed. Besides protons and neutrons, highly reactive and biologically hazadrous fragments are produced (e.g. high energetic $\alpha$-particles), even more in higher $\mathrm{Z}$ target materials or in heavy constituents of the GCR.

The secondaries produced by target and projectile fragmentation continue to traverse the volume of the spacecraft and may themselves undergo further 
nuclear reactions. Conclusively, the attenuation of ionizing radiation and the production of secondary particles is inherently related to the attributes of materials chosen for primary structure, insulation, meteoroid protection systems, onboard equipment and supplies. The transport of primary ionizing radiation through the spacecraft structure and formation of secondary particles are summarized in figure 3 .

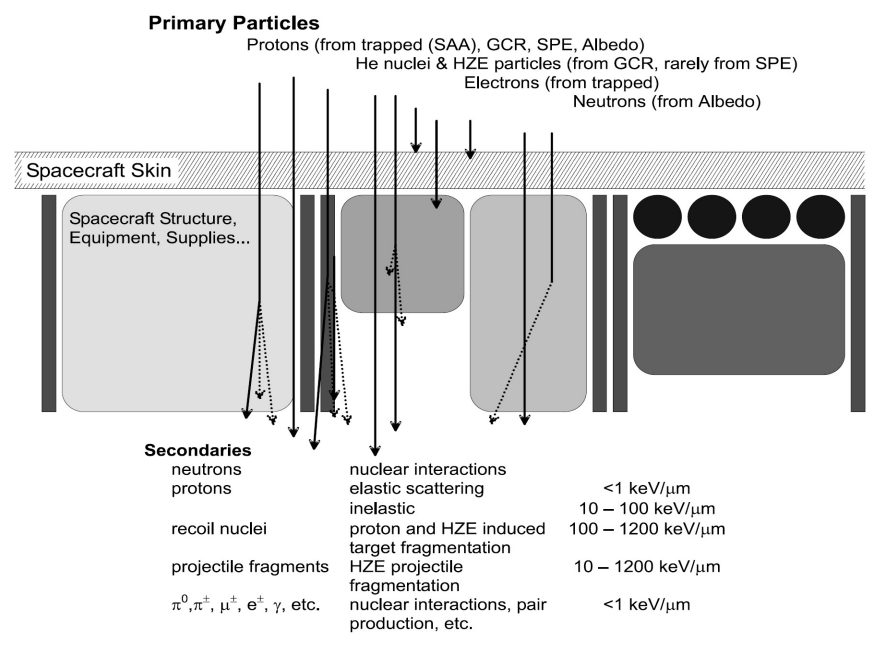

Figure 3: Transport of primary ionizing radiation through the spacecraft structure and the generation of secondary particles [8]

\subsection{Biological Responses and Health Risks}

Effects of ionizing radiation on the human body are commonly separated into deterministic effects (short term) and stochastic effects (long term). Deterministic effects from the intense radiation dose received from a SPE within a few hours to days may cause skin damage, some hematological responses including blood count changes, immune failure and possibly nausea [4. Those effects are partly reversible. Stochastic effects are referred to as cancer, tumor formation and neurological disorders, identified many years after the exposure. For stochastic effects, an increase of $3 \%$ for death by cancer is the risk criterion for the development of exposure limits (referred to in chapter 4.1).

The biological outcome caused by radiation dose deposited in living tissue, cells and DNA is called the biological response. Determining those responses to ionizing radiation is complex, explicitely for long-term exposure and varying particle species. HZE ions have a significantly larger effect on human cells due to their relatively low LET in comparison to lighter ions. The energy deposition is roughly proportional to the square of the atomic number. An equal amount of deployed dose would require several hundred protons, so the passing of a single high energetic Fe ion would be a devastating event [9].

\section{Simulation \& Radiation Trans- port Codes}

\subsection{Dosimetric Values}

The described health risks are characterized by the following macroscopic quantities used in radiation protection [9]:

Absorbed dose $D=\frac{d \bar{\epsilon}}{d m}$ is a measure of the mean energy deposited per unit mass of medium by ionizing radiation and has the unit $\mathrm{J} / \mathrm{kg}$, which is given the special name Gray (Gy), $1 \mathrm{~Gy}=100 \mathrm{rad}$. However, this quantity does not take into account the biological effectiveness of different ionizing particles. For valuation of radiation quality and risk assessment, the so called dimensionless quality factor $\mathrm{Q}$ was introduced. $\mathrm{Q}$ values in dependence of the LET, recommended by the ICRP 1 are given in table 2, The dose equivalent $\mathbf{H}$ was defined by ICRP as an operational quantity and is calculated by multiplying the absorbed dose $\mathrm{D}$ with the quality factor Q:

$$
H=Q \cdot D
$$

\begin{tabular}{cccc}
\hline \multicolumn{2}{c}{ ICRP 26} & \multicolumn{2}{c}{ ICRP 60} \\
$\mathrm{~L}_{\infty}(\mathrm{keV} / \mu \mathrm{m})$ & $\mathrm{Q}(\mathrm{LET})$ & $\mathrm{L}_{\infty}(\mathrm{keV} / \mu \mathrm{m})$ & $\mathrm{Q}(\mathrm{LET})$ \\
\hline$\leq 3.5$ & 1 & $\leq 10$ & 1 \\
7 & 2 & $10-100$ & $0.32 L-2.2$ \\
23 & 5 & $\leq 10$ & $300 / \sqrt{L}$ \\
53 & 10 & & \\
175 & 20 & & \\
\hline
\end{tabular}

Table 2: Q values in dependence of LET, ICRP 26 (1977) [10] and ICRP 60 (1991) [1] recommendations

\footnotetext{
${ }^{1}$ International Commission on Radiological Protection, Stockholm, Sweden
} 
This concept is still used in radiation transport codes and will be primarily addressed within this study, although the ICRP has partly revised it by introducing the radiation weighting factor $\mathbf{w}_{\mathbf{R}}$ in replacement of $\mathrm{Q}$. The factor depending on particle type and energy can be seen in table 3 .

\begin{tabular}{|c|c|}
\hline $\begin{array}{l}\text { Type and } \\
\text { energy range }\end{array}$ & $\begin{array}{l}\text { Radiation weighting } \\
\text { factor } w_{R}\end{array}$ \\
\hline $\begin{array}{l}\text { Photons, } \\
\text { all energies }\end{array}$ & 1 \\
\hline $\begin{array}{l}\text { Electrons and muons, } \\
\text { all energies }\end{array}$ & 1 \\
\hline Neutrons, energy & 5 \\
\hline $10 \mathrm{keV}$ to $100 \mathrm{KeV}$ & 10 \\
\hline$>100 \mathrm{keV}$ to $2 \mathrm{MeV}$ & 20 \\
\hline$>20 \mathrm{MeV}$ & 10 \\
\hline $\begin{array}{l}\text { Protons, energy }>2 \mathrm{MeV} \\
\alpha \text { particles, fission fragments, } \\
\text { heavy nuclei }\end{array}$ & 20 \\
\hline
\end{tabular}

Table 3: Radiation weighting factors $w_{R}$ for different particle types and energies 11

The probability of stochastic effects from radiation is found to vary with the organ or tissue irradiated. Therefore, the tissue weighting factor $\mathbf{w}_{\mathbf{T}}$ was introduced, which is used with the organ equivalent dose to derive the newly defined effective dose $\mathbf{E}$ :

$$
E=\sum_{T} w_{T} \sum_{R} w_{R} \cdot D_{T, R}
$$

Values for $w_{T}$ reach from 0.01 for skin tissue to 0.12 for bone marrow and 0.20 for gonads and include all organs likely to be selectively irradiated and/or known to be susceptible to cancer induction.

These newer conceptual quantities for radiation dose assessment have not yet been implemented into the radiation transport codes utilized in this study. The effective dose is not measureable within the human body and moreover, difficult to be included into the radiation algorithms. Its quantitative assessment demands ray tracking of all secondaries (and maybe tertiaries) produced by a primary particle in a specific tissue site within a virtual human body (CAM) in order to weight them with the $w_{R}$ of the first particle entering the tissue. This is necessary to calculate the whole dose $D_{T, R}$ in a tissue $\mathrm{T}$, which may originate from a radiation $\mathrm{R}$ first entering the tissue site, but deployed by many secondary particles produced when this particle traverses the tissue volume. This complex operation is difficult to be converted into a numerical calculation of effective dose $\mathrm{E}$ and is subject of ongoing efforts.

The NCRP, the National Council on Radiation Protection and Measurements (USA) has published limit recommendations for ionizing radiation exposure during operational missions in LEO. Throughout all manned missions in earth orbit, primarily onboard the ISS, these recommendations apply on the basis not to exceed the lifetime fatal cancer risk projection by $3 \%$ (table 4 ). The limits refer to the most sensitive parts of the human body: skin, ocular lens (eye) and blood forming organs (BFO) [12].

\begin{tabular}{lccc}
\hline \multicolumn{4}{l}{ Organ dose equivalent $\mathrm{H}[\mathrm{Sv}]$} \\
\hline organ & BFO & Eye & Skin \\
\hline Career & see Table 5 & 4.0 & 6.0 \\
Annual & 0.50 & 2.0 & 3.0 \\
30 days & 0.25 & 1.0 & 1.5 \\
\hline
\end{tabular}

Table 4: NCRP Report 98 (1989) [13] and NCRP Report 132 (2000) [14 recommendations for organ dose equivalent limits during operations in LEO

\begin{tabular}{lcccc}
\hline \multicolumn{4}{l}{ Age specific whole-body career } & dose limits $[\mathrm{Sv}]$ \\
\hline Age & 25 & 35 & 45 & 55 \\
\hline Female & 0.4 & 0.6 & 0.9 & 1.7 \\
Male & 0.7 & 1.0 & 1.5 & 3.0 \\
\hline
\end{tabular}

Table 5: NCRP (2000) recommendations for ten year career whole-body dose limits during operations in LEO (based on $3 \%$ lifetime risk of induced cancer) [12]

These limits apply only to crews in LEO and are not to be considered as appropriate limits or guidance for deep space missions. Guidance for missions beyond LEO do not currently exist [12. According to the NCRP, this is a consequence of the large uncertainties in predicting the risks of stochastic (late) effects from heavy cosmic ions. 


\subsection{Computational Approach}

\section{Deterministic Codes, HZETRN}

The numerical calculations done in this study as a first qualitative approach were primarily done using the deterministic radiation transport code HZETRN2005 from NASA Langley Research Center (LaRC). It has been developed to evaluate the radiation fields within sensitive materials, electronic devices and human tissue behind materials in space [15]. The code is applicable to protons, neutrons and multiple charged ions in the radiation environment. HZETRN calculates the transport of primary ions and their secondary particles and fragments and thus give a complete physical description in a one dimensional approach, which means secondaries created in the shield only move along the initial path of the primary particle (straight-ahead approximation).

\section{Transport Theory}

The types and energy distributions of particles transmitted through a shield material requires the solution to the coupled linear Boltzmann transport equations with boundary conditions related to the external space radiation environment. These transport equations for energetic particles are obtained by balancing the change in particle flux as they cross a small volume of material with the gains and losses caused by nuclear collisions (conservation principle) [2]. It considers a spherical region of space filled with matter described by appropriate atomic and nuclear cross sections. With the flux density (particles/cm2-sr-s-MeV/n) $\phi_{j}(\vec{x}, \vec{\Omega}, E)$ at position $\vec{x}$ for particle type j moving in direction $\vec{\Omega}$ with energy E as:

$$
\begin{aligned}
& \vec{\Omega} \cdot \nabla \phi_{j}(\vec{x}, \vec{\Omega}, E)=\sum \int_{k} \sigma_{j k}\left(\vec{\Omega}, \overrightarrow{\Omega^{\prime}}, E, E^{\prime}\right) \\
& \cdot \phi_{k}\left(\vec{x}, \overrightarrow{\Omega^{\prime}}, E^{\prime}\right) d \overrightarrow{\Omega^{\prime}} d E^{\prime}-\sigma_{j}(E) \cdot \phi_{j}(\vec{x}, \vec{\Omega}, E)
\end{aligned}
$$

where $\vec{x}$ is a vector to the center of the sphere, $\vec{\Omega}$ is normal to the surface element, $\mathrm{E}$ is the particle energy and $\sigma_{j}(E)$ and $\sigma_{j k}\left(\vec{\Omega}, \overrightarrow{\Omega^{\prime}}, E, E^{\prime}\right)$ are the shield medium's macroscopic cross sections. The $\sigma_{j k}\left(\vec{\Omega}, \overrightarrow{\Omega^{\prime}}, E, E^{\prime}\right)$ represents all those processes by which type $\mathrm{k}$ particles moving in direction $\overrightarrow{\Omega^{\prime}}$ with energy E' produce a type j particle in direction $\vec{\Omega}$ with energy E. The term 'cross section' refers to the probability of the respective reaction occuring between the projectile particle and the target atoms [16]. For further details refer to the quoted publications.

\section{Monte Carlo codes, MULASSIS}

The Geant4 (GEometry ANd Tracking) based multilayered shielding simulation software tool (Mulassis) was developed as part of the European Space Agency (ESA) activities in the Geant4 collaboration. It was derived from the Geant4 Monte Carlo (M-C) simulation toolkit for the passage of particles through matter, developed by a large international collaboration of scientists and software engineers lead by CERN2 2 . Despite utilization in nulcear, medical and accelerator physics sciences, this new generation of radiation transport codes provides full three-dimensional treatment of the wide range of particle species and interaction processes and is therefore applicable also to the radiation environment [3]. The toolkit allows treatment of particles from thermal to $\mathrm{PeV}$ energies and currently includes implementation of physics like electromagnetic ionization, multiple scattering, Bremsstrahlung, photo-electric effect, Compton scattering, pair-production and atomic relaxation (among others). The application program Mulassis has been developed based on Geant4, which then is capable of being used by spacecraft design engineers without the need of additional programming.

The Mulassis tool is applicable for dose, dose equivalent and fluence analysis behind various shields and materials. The user can define the shielding/detector geometry as planar or spherical layers, with the materials defined by their density and elemental/isotopic composition.

\section{Modelling of the Human Body}

A very substantial but in same degree complicated issue is the modelling of the human body as the target of concern after the shield. The ICRP rec-

\footnotetext{
${ }^{2}$ Conseil Européen pour la Recherche Nucléaire, European Organization for Nuclear Research, Geneva, France/Switzerland
} 
ommended radiation dose limits for the most sentitive organs within the body like the blood forming organs $(\mathrm{BFO})$, the ocular lens, skin and various other organs. The tissue composition and densities of these body parts are varying and so are the recommended tissue weighting factors for dose estimation (see Chapter 4.1). Furthermore, the body self-shielding through outward lying tissue sections and the exact location of the organs towards the particle trajectories have to be taken into account. Only with a significant emphasis on these aspects, 'reality' can be pictured adequatly. In order to meet those requirements, the so called 'Computerized Anatomical Man' (CAM) and Female (CAF) models [17] have been implemented. These models include a detailed mapping of an average human body with organ location, dimension and tissue density definitions. Nevertheless, they are not implemented within the publicly available radiation codes and thus their utilization for computational radiation dose assessment is not possible at the moment.

\section{Case Study for a Human Mis- sion to Mars}

Since this study concentrates on protection from cosmic radiation during an exploratory mission in deep space and not on the developement of appropriate mission scenarios, the reference scenarios for this case study have been selected from the NASA reference mission to Mars [1]. The selected mission scenarios are listed in table 6 .

\begin{tabular}{ll}
\hline Scenario 1 & $\approx 900$ day total, long term stay on Mars \\
\hline Scenario 2 & $\approx 500$ day total, short term stay on Mars \\
\hline Scenario $3 \approx 900$ day fast-transit, long term stay \\
\hline
\end{tabular}

Table 6: Chosen Mission Scenarios [1]

The trajectories and segment dates are shown exemplarily in figure 4. Although the mentioned flight dates are no longer feasible within current NASA plans for deep space exploration, the transit times are also representative for missions in different epoches.

The considered 'Scenario 2' with a short term stay on Mars has higher propulsive requirements than the long term 'Scenario 1' and typically requires a gravity-assisted swingby at Venus or the performance of a deep-space propulsive maneuver to reduce total mission energy and constrain Mars and Earth reentry speeds. Other disadvantages arise from generally longer transfer times and thus stay of astronauts in free space (approx. $90 \%$ of total mission time) and from the requirement of a close passage by the sun (0.7 AU or less), which gives rise to the risks from even higher fluxes received from an SPE.

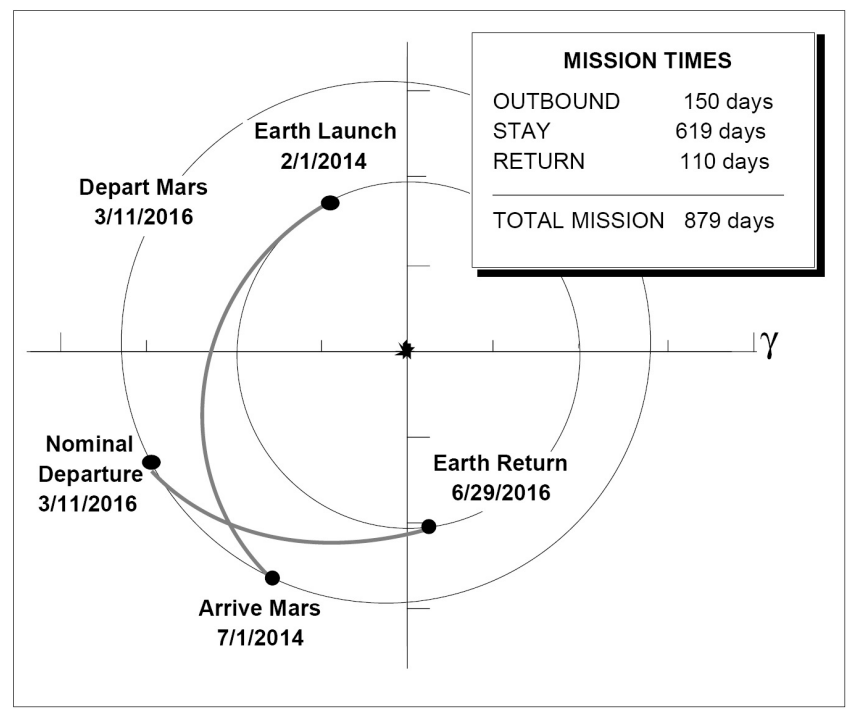

Figure 4: Typical Trajectories for Scenario 3, Fast Transit Mission 1

The details of the chosen mission and segment durations can be seen in table 7 .

\begin{tabular}{lccc}
\hline & \multicolumn{3}{c}{ Duration (days) } \\
\hline Mission Phase & Scenario 1 & Scenario 2 & Scenario 3 \\
\hline Earth-Mars Transfer & 225 & 224 & 150 \\
Stay on Mars & 458 & 30 & 619 \\
Mars-Earth Transfer & 237 & 291 & 110 \\
\hline Total mission & 919 & 545 & 879 \\
\hline
\end{tabular}

Table 7: Mission segments duration [1]

\subsection{Materials for Radiation Protection}

The basic principle behind passive protection from cosmic particles with materials is their hydrogen content. Unless our intuitive understanding about shielding from radiation, there is an inherent difference in comparison to electromagnetic radiation as $\mathrm{X}$ - and $\gamma$-rays, commonly known from medical 
checkups and atomic reactors. For those radiation types, shielding with heavy shield matter like lead, tungsten or concrete is appropriate. As depicted above, the cosmic environment predominantly includes particle radiation, the desired materials thus must allow a high electronic energy loss, while at the same time decrease the probability of nuclear fragmentation processes (cf. chapter 3). While electronic energy loss depends on the number of electrons whereas nuclear interactions depend on the number of nucleons, the best shielding materials must possess the highest ratio of electrons to nucleons. Hydrogen, with exactly one electron and a one-proton nucleus, has the highest ratio of any known element. Therefore, hydrogenic materials are essential for passive radiation shielding [18].

The examined materials are hydrogenated graphite nanofibers (HGNF), lithium hydride $(\mathrm{LiH})$, polyethylene (PE), polysulfone (PSO), polyetherimide (PEI), water $\left(\mathrm{H}_{2} \mathrm{O}\right)$ and aluminium (AL2024). Al2024 alloy is almost equal to the more frequently used Al2219 alloy for spacecrafts and onboard ISS. Additional materials and matters as liquid hydrogen $\left(\mathrm{LH}_{2}\right)$, liquid methane $\left(\mathrm{CH}_{2}\right)$ and carbon fiber reinforced plastic (CFRP) are currently under investigation and results are pending.

NASA currently investigates on polyethylene- and graphite-fiber reinforced composites, originally intended as a ballistic shield.

\begin{tabular}{lc}
\hline \multicolumn{2}{c}{ Hydrogen content of selected materials } \\
\hline Material & Number of $\mathrm{H}$ atoms per $\mathrm{cm}^{3}$ \\
\hline Solid Hydrogen $(4.2 \mathrm{~K})$ & 5.7 \\
Water & 6.7 \\
Lithium hydride $(\mathrm{LiH})$ & 5.9 \\
Pure polyethylene $(\mathrm{PE})$ & 5.9 \\
\hline
\end{tabular}

Table 8: Hydrogen content of selected materials 19]

PE fibers have excellent physical properties, including the highest specific strength of any known material [19]. These bricks have a fabric layer shape and weigh almost half as much as aluminium. Epoxy is widely used as matrix resins in advanced composite systems. These resins also have substantial hydrogen content, making them suitable candidate materials for radiation shielding. The hydrogen content of selected materials are given in table 8 . The variability of fiber-matrix combinations even qualifies this composite to serve a structural function [20]. Use of composite structures for aircrafts is steadiliy increasing, $50 \%$ of the structural weight of the new Boeing 787, including its fuselage, is in carbon-based and similar composites. Specific attributes are not yet available for these advanced materials, thus they are not accounted for in this study.

\subsection{Simulation Setup - Preliminary Re- sults}

Within our first approach presented in this paper, the dose $\mathrm{D}$ and dose equivalent $\mathrm{H}$ in a thin detecting tissue layer directly behind a spherical shield of three meter radius have been calculated utilizing the two described radiation transport codes. This provides a substantial preliminary validation between the two codes and will be the offset for future calculations. The input radiation spectra for GCR have been taken from the Badhwar-O'Neill model 2005 [21], a highly accurate analytical set of formula for each HZE species (from hydrogen to nickel) within the GCR.

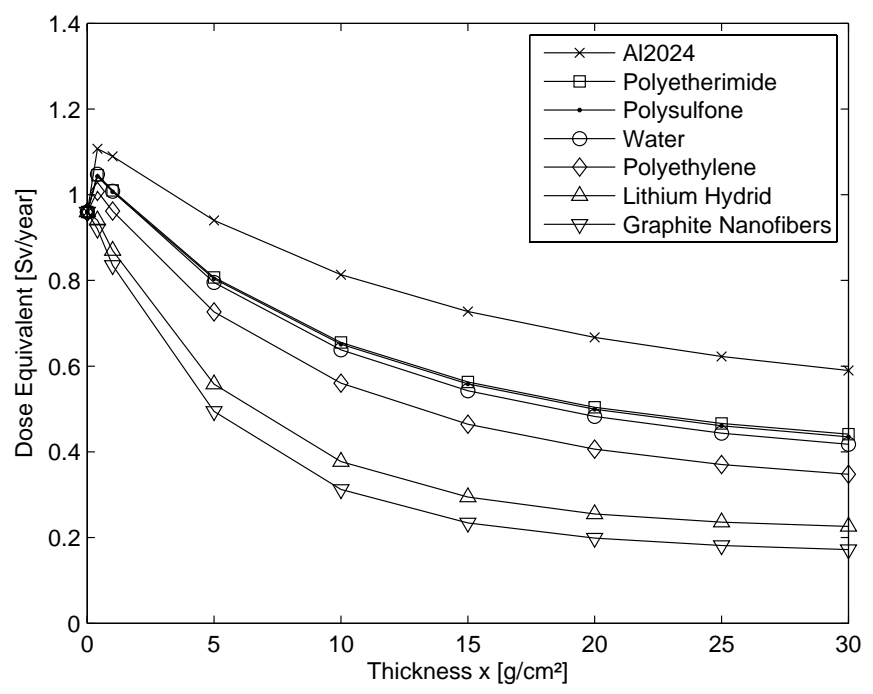

Figure 5: Yearly Dose Equivalent H for GCR during transfer at solar minimum in $\mathrm{Sv} /$ year for different materials calculated with HZETRN

The proton spectrum for the analyzed large August 1972 SPE was taken from the King model [22].

The dose equivalent from GCR during transfer calculated with HZETRN for a variety of different ma- 


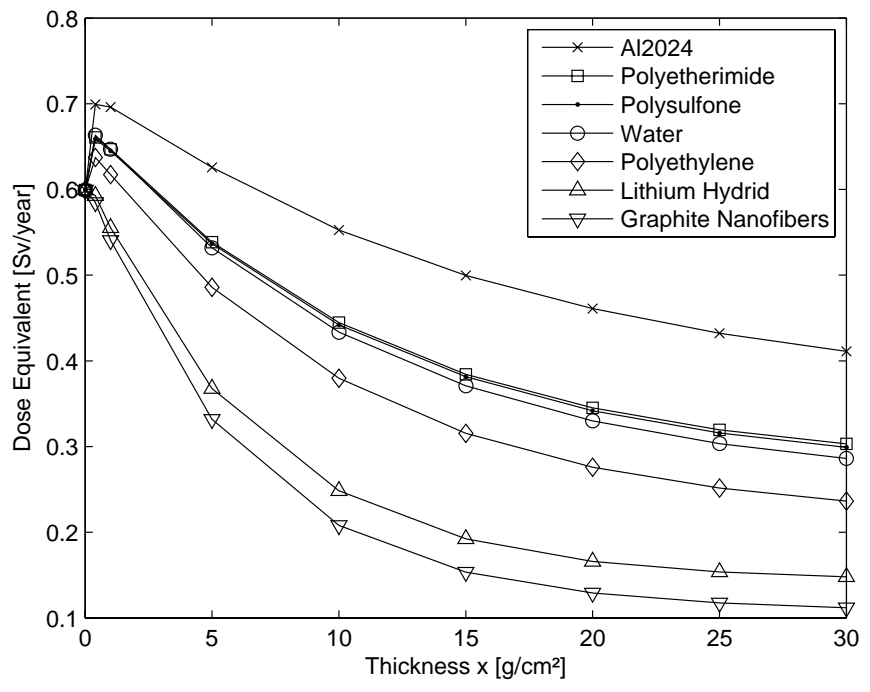

Figure 6: Yearly Dose Equivalent H for GCR during transfer at solar maximum in $\mathrm{Sv} /$ year for different materials calculated with HZETRN

terials at solar minimum and maximum are shown in figure 5 and figure 6 . An exponentially decreasing behavior with rising shield thickness is visible after an initial peek at very low shield thicknesses. The origin of this peek is very essential for passive radiation protection through materials: Any matter of higher atomic number causes the incident cosmic particles to emit low energy neutrons (and protons) due to nuclear collisions and recoil processes. Only very hydrogen-rich materials like $\mathrm{LiH}$ and (potentially) HGNF do not show this peek. Here, the primary particles merely find the electron clouds around the shield's atoms instead of colliding with a nucleus. The figures clearly show this advantage with increasing hydrogen content: the tested materials reach the same level of protection at about $80 \%$ (PEI, PSU), $78 \%$ (water), $69 \%$ (PE), $46 \%(\mathrm{LiH})$ and $38 \%(\mathrm{GNF})$ areal density (thickness) in comparison to aluminum.

The same setup of materials calculated with the August 1972 SPE is visible in figure 7. Again the exponential decrease is visible and is even stronger in comparison to the GCR.

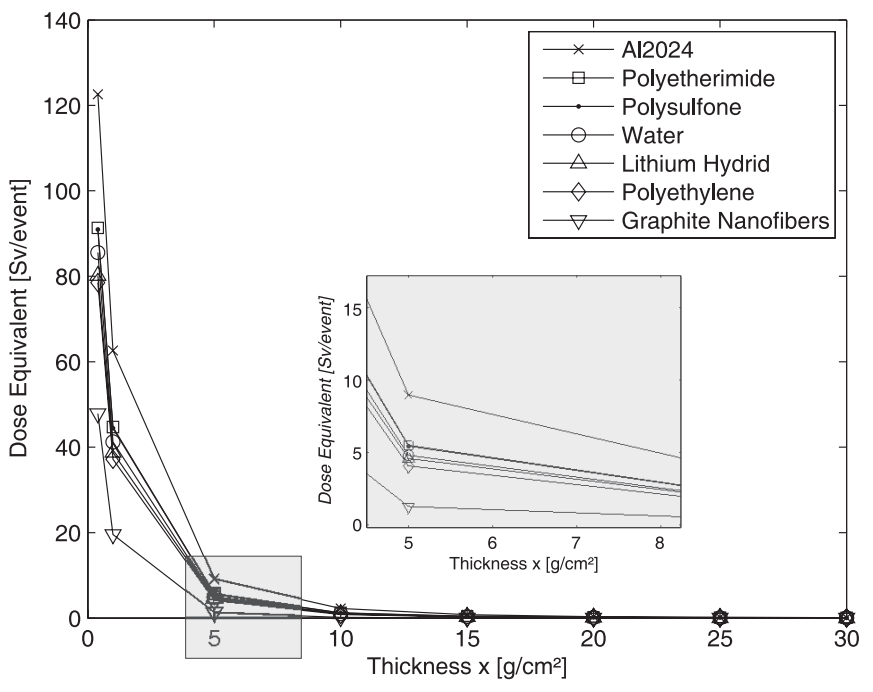

Figure 7: Aug 1972 SPE dose equivalent H per whole event calculated with HZETRN

This is due to the character of a solar particle event and becomes visible by conferring its energy spectrum in figure 2 with the GCR spectra in figure 1. The particle energies during an SPE hardly ever exceed $600 \mathrm{MeV}$ but show a particle flux of about 6 magnitudes higher than for GCR. This gives the SPE its devastating character: about hundred million particles with moderate energies (compared to GCR) are hitting the spacecraft per $\mathrm{cm}^{2}$ within the time frame of several hours during a possible 'worst case' event.

Nevertheless, the particle flux is rapidly decreasing for higher energies. Conclusively, when shield thickness reaches a certain amount, the majority of particles will be decelerated within the shield, their quantity is irrelevant. GNF, $\mathrm{LiH}$ and $\mathrm{PE}$ again show best attenuation, whereas PE even succeeds $\mathrm{LiH}$ in comparison to shield effectiveness against galactic cosmic radiation. In relation to the $\mathrm{H}$ value behind $10 \mathrm{~g} / \mathrm{cm}^{2} \mathrm{Al}$, GNF reaches same level of protection at about $47 \%$ areal density (thickness), $\mathrm{PE}$ at $77 \%$ and $\mathrm{LiH}$ at $83 \%$.

Figure 8 shows the same August 1972 SPE spectrum calculated through an Al shield with Mulassis in comparison to HZETRN. The gradients of $\mathrm{H}$ in a thin tissue target directly behind the shield are in good aggreement. Accordingly, the comparison for GCR at solar minimum conditions calculated with both codes is given in figure 9. Here the picture is a little different: The curves show similar progressions, but the dose equivalent calculated by 
Mulassis for the same tissue target setup falls off to about $60 \%$ in contrast to HZETRN. This might be due to variations in the utilized input spectra for GCR and remains to be analyzed in detail. The similar progression for increasing $\mathrm{Al}$ shield thickness validates the principal conformity between the two codes (Monte Carlo and deterministic) and the atomic and nuclear physics implemented.

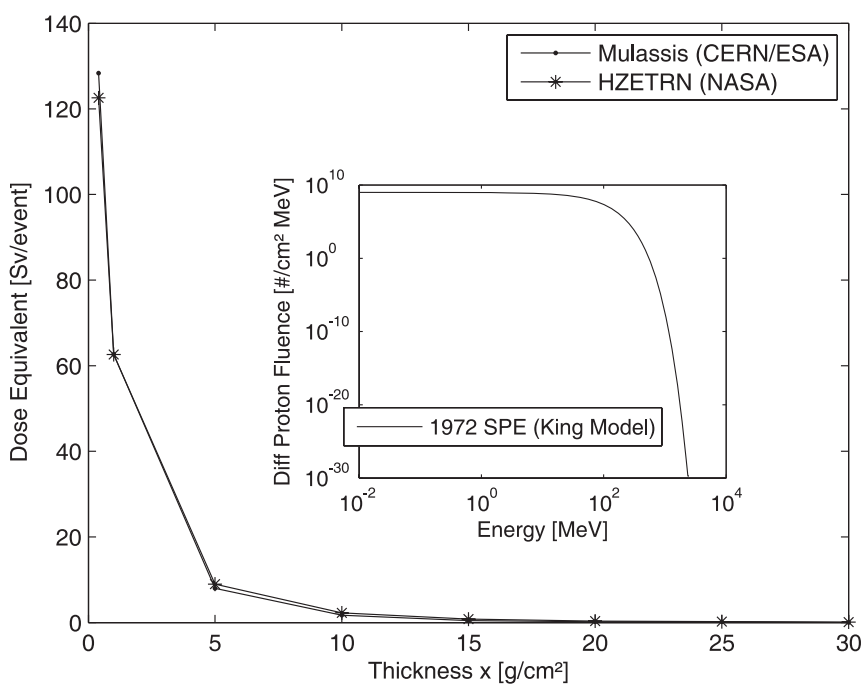

Figure 8: Comparison between HZETRN and MULASSIS for Aug 1972 SPE and SPE spectrum

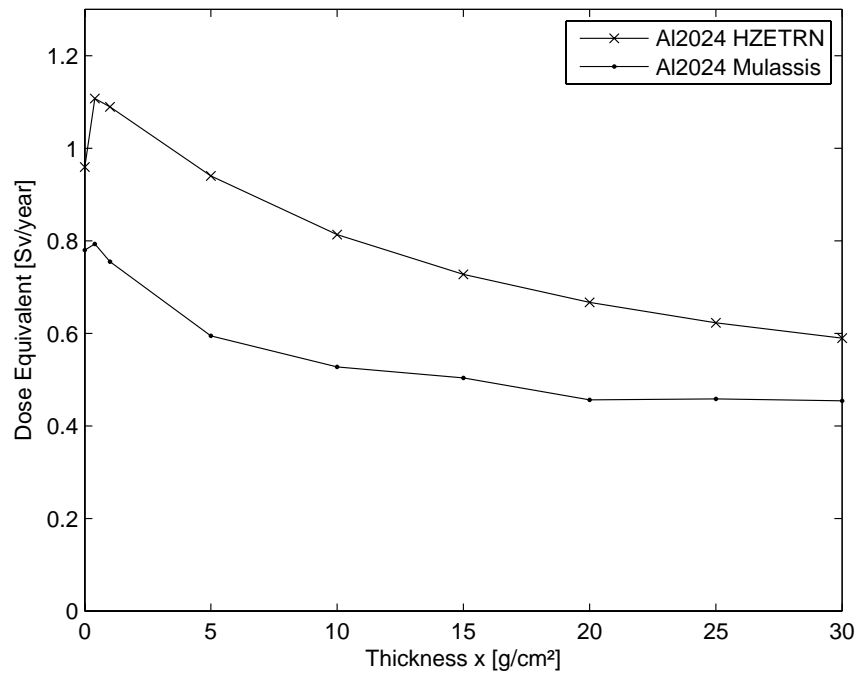

Figure 9: Comparison between HZETRN and MULASSIS for GCR at solar minimum spectra behind Al2024 shield

When combining different materials into a multi layered shield configuration, the radiation dose received by an astronaut can be further reduced.

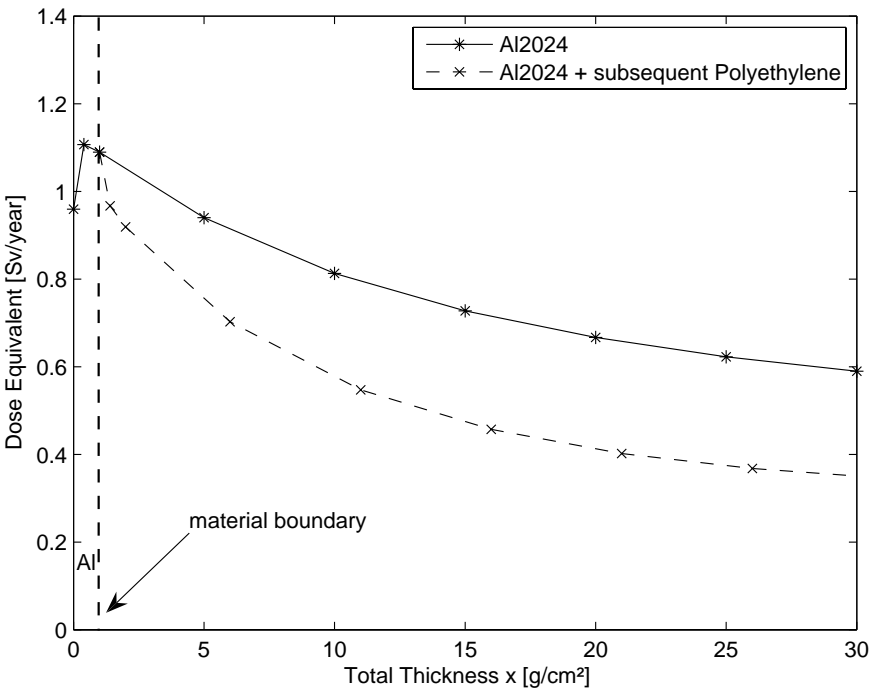

Figure 10: Comparison of Al2024 alloy and a dual layer configuration of $4 \mathrm{~mm} \mathrm{Al}$ and subsequent PE calculated with HZETRN

Assuming a regular pressure shell of $1 \mathrm{~g} / \mathrm{cm}^{2} \mathrm{Al}$ (about $4 \mathrm{~mm}$ ) and subsequent PE bricks, this may enhance the shield performance significantly as seen in figure 10. A detailed analysis of the Mars radiation environment and the performance of the chosen materials on the martian surface will be topic of ongoing investigations. The $\mathrm{BFO}$ dose equivalent in $\mathrm{rem} / \mathrm{yr}(100 \mathrm{rem}=1 \mathrm{~Sv})$ in dependence of $\mathrm{CO}_{2}$ shield thickness is visible in figure 11 . Very significant is the reaction of heavy ions with the atmosphere through production of secondary particles (protons, neutrons) in nuclear fragmentation processes. The dose from primary HZE ions is hence reduced significantly for higher atmospheric areal densities and thus on ground level, the secondaries deliver the greater percentage of the dose. This shielding effect depends on the atmospherical density varying between 16 and 22 g $\mathrm{CO}_{2} / \mathrm{cm}^{2}$ (see dashed lines in the figure) [5].

\subsection{Risk Estimation For Case Study Missions - Reference Results}

The calculation of reliable values for dose equivalent $\mathrm{H}$ within the human body during an interplanetary mission to Mars has shown to be infeasible with the currently available radiation programs without implementation of a detailed modellation of the human body. Therefore, preliminary results 


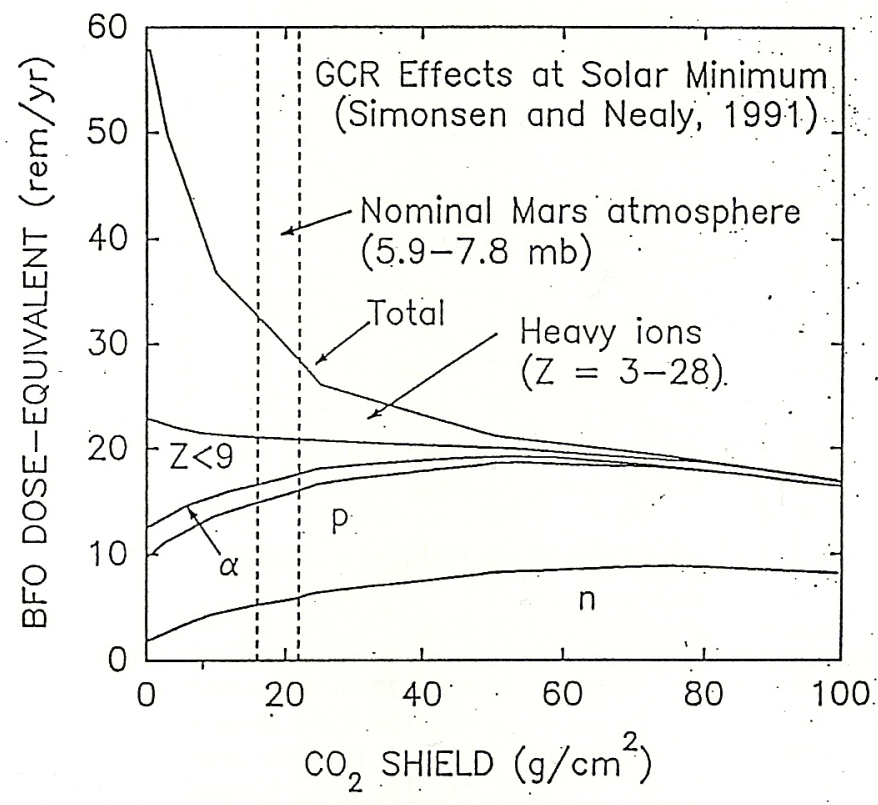

Figure 11: BFO dose equivalent behind $\mathrm{CO}_{2}$ shielding and nominal Mars atmosphere

from radiobiological papers have be chosen to evaluate the total dose assumption during the chosen mission scenarios. As reference, papers from Wilson [23], Simonsen [24] and Saganti [25] have been chosen and are summarized in tables 9 and 10 for interplanetary transfer and martian surface stay.

\begin{tabular}{|c|c|c|c|c|}
\hline \multicolumn{5}{|c|}{ Organ dose Equivalent H from GCR [Sv/year] } \\
\hline \multirow[t]{2}{*}{ Shield (thickness) } & \multicolumn{4}{|c|}{ organ } \\
\hline & $\mathrm{BFO}$ & Skin & Eye & Point \\
\hline \multicolumn{5}{|c|}{ Mars surface, Point estimate of BFO dose } \\
\hline & \multicolumn{2}{|c|}{ Solar min } & \multicolumn{2}{|c|}{ Solar max } \\
\hline at $0 \mathrm{~km}$ & \multicolumn{2}{|c|}{$0.105-0.119$} & \multicolumn{2}{|c|}{$0.057-0.061$} \\
\hline at $4 \mathrm{~km}$ & \multicolumn{2}{|c|}{$0.120-0.138$} & \multicolumn{2}{|c|}{$0.062-0.068$} \\
\hline
\end{tabular}

Table 9: Summary of annual dose equivalent $H$ from GCR and SPE behind various shields on Mars surface from current radiobiological studies

The total mission dose assumption has been performed for both solar maximum and minimum, utilizing the three mission scenarios and two structural design setups as a baseline (see table 11). Setup 1 represents the current $1 \mathrm{~g} / \mathrm{cm}^{2} \mathrm{Al}(\approx$ $4 \mathrm{~mm}$ ) pressure shell of an ISS module without MDPS 3 , fixtures or equipment installed. Setup

\begin{tabular}{|c|c|c|c|}
\hline \multicolumn{4}{|c|}{ Organ dose Equivalent H from GCR [Sv/year] } \\
\hline Shield thickness & & & \\
\hline & $\mathrm{BFO}$ & Skin & Eye \\
\hline Aluminium & \multicolumn{3}{|c|}{ Transfer, 1977 solar min } \\
\hline unshielded & 0.731 & 0.961 & 1.043 \\
\hline $1 \mathrm{~g} / \mathrm{cm} 2$ & 0.712 & 0.988 & 0.975 \\
\hline $3 \mathrm{~g} / \mathrm{cm} 2$ & 0.677 & 0.921 & 0.905 \\
\hline $5 \mathrm{~g} / \mathrm{cm} 2$ & 0.647 & 0.863 & 0.846 \\
\hline $10 \mathrm{~g} / \mathrm{cm} 2$ & 0.589 & 0.754 & 0.735 \\
\hline Aluminium & \multicolumn{3}{|c|}{ Transfer, 1970 solar max } \\
\hline unshielded & 0.277 & 0.327 & 0.348 \\
\hline $1 \mathrm{~g} / \mathrm{cm} 2$ & 0.272 & 0.348 & 0.344 \\
\hline $3 \mathrm{~g} / \mathrm{cm} 2$ & 0.263 & 0.337 & 0.332 \\
\hline $5 \mathrm{~g} / \mathrm{cm} 2$ & 0.256 & 0.325 & 0.319 \\
\hline $10 \mathrm{~g} / \mathrm{cm} 2$ & 0.239 & 0.299 & 0.292 \\
\hline Aluminium & \multicolumn{3}{|c|}{ Transfer, Aug 1972 SPE [Sv/event] } \\
\hline $0.4 \mathrm{~g} / \mathrm{cm} 2$ & 2.170 & 93.50 & 38.30 \\
\hline $1 \mathrm{~g} / \mathrm{cm} 2$ & 1.800 & 35.60 & 21.40 \\
\hline $5 \mathrm{~g} / \mathrm{cm} 2$ & 0.650 & 4.270 & 3.670 \\
\hline $10 \mathrm{~g} / \mathrm{cm} 2$ & 0.243 & 1.100 & 1.010 \\
\hline $25 \mathrm{~g} / \mathrm{cm} 2$ & 0.059 & 0.168 & 0.168 \\
\hline Polyethylene & \multicolumn{3}{|c|}{ Transfer, 1977 solar min } \\
\hline unshielded & 0.731 & 0.961 & 1.043 \\
\hline $1 \mathrm{~g} / \mathrm{cm} 2$ & 0.695 & 0.922 & 0.947 \\
\hline $3 \mathrm{~g} / \mathrm{cm} 2$ & 0.633 & 0.806 & 0.830 \\
\hline $5 \mathrm{~g} / \mathrm{cm} 2$ & 0.584 & 0.717 & 0.741 \\
\hline $10 \mathrm{~g} / \mathrm{cm} 2$ & 0.499 & 0.568 & 0.591 \\
\hline Polyethylene & \multicolumn{3}{|c|}{ Transfer, 1970 solar max } \\
\hline unshielded & 0.277 & 0.327 & 0.348 \\
\hline $1 \mathrm{~g} / \mathrm{cm} 2$ & 0.265 & 0.324 & 0.334 \\
\hline $3 \mathrm{~g} / \mathrm{cm} 2$ & 0.246 & 0.295 & 0.304 \\
\hline $5 \mathrm{~g} / \mathrm{cm} 2$ & 0.229 & 0.269 & 0.278 \\
\hline $10 \mathrm{~g} / \mathrm{cm} 2$ & 0.199 & 0.222 & 0.229 \\
\hline Polyethylene & \multicolumn{3}{|c|}{ Transfer, Aug 1972 SPE [Sv/event] } \\
\hline $0.4 \mathrm{~g} / \mathrm{cm} 2$ & 2.210 & 67.70 & 35.30 \\
\hline $1 \mathrm{~g} / \mathrm{cm} 2$ & 1.740 & 25.10 & 18.10 \\
\hline $5 \mathrm{~g} / \mathrm{cm} 2$ & 0.500 & 2.670 & 2.510 \\
\hline $10 \mathrm{~g} / \mathrm{cm} 2$ & 0.155 & 0.580 & 0.569 \\
\hline $25 \mathrm{~g} / \mathrm{cm} 2$ & 0.017 & 0.035 & 0.037 \\
\hline
\end{tabular}

Table 10: Summary of annual dose equivalent H from GCR and SPE behind various shields during transfer from current radiobiological studies

\footnotetext{
${ }^{3}$ Meteoroid and Debris Protection System
} 
2 extends the basic setup 1 with subsequent $\mathrm{PE}$ bricks of $10 \mathrm{~g} / \mathrm{cm}^{2}(\approx 11 \mathrm{~cm})$, installed after the primary structure.

For solar maximum, the possible occurence of a solar particle event such as the hazadrous August 1972 SPE has been considered. A stay behind a $10 \mathrm{~g} / \mathrm{cm}^{2}$ shelter during hours of the solar storm has been considered in comparison to no additional shielding. It is assumed that the astronauts will be warned timely from mission control on earth or will utilize onboard warning detectors and therefore spend the whole time during an SPE within the shelter. Also included in the table are the percentaged relations to the recommended annual and career dose limits. As the total career dose is recommended as age and gender specific, calculation is performed for both sexes and for 45 year old astronauts 4 . The dose delivered to the vital organs is the most important with regard to latent carcinogenic effects. This dose is often taken as the whole-body exposure (cf. table 5) and is assumed almost equal to the blood-forming organ (BFO) dose [26]. The dose on martian surface is assumed behind nominal habitat shielding [24], since no detailed calculations are performed and/or publicly available at present.

The calculations show a considerable advantage for the (fast transit) Scenario 3: Minimizing transfer time reduces the total mission dose to about $70 \%$ (during solar min.) and $80 \%$ (solar max.) in comparison to Scenario 1, certifying the martian atmosphere's protective character. There is a wide discussion about when the mission shall take place during the solar cycle. The reference analysis points out an average reduction of dose equivalent of approx. $65 \%$ when travelling during solar maximum conditions. This results do not account for the possibility of several large SPEs and assumes full storm shielding during the hours (or maybe days) of a solar eruption. Annual limit recommendations will be exceeded by almost all configurations except for setup 2 at solar min., whereas the astronauts stay within their 10-year career dose limit in most cases. Here again shall be alluded to the original definition of exposure limit recommendations for LEO operations only and their limited application for interplanetary missions.

\footnotetext{
${ }^{4}$ The author assumes a beneficial impact on mission goal achievement when accomplished by middle-aged astronauts
}

Total mission dose eq. [Sv] to BFO (Solar min)

Scenario $1 \quad$ Scenario $2 \quad$ Scenario 3

Setup 1: S/C with $1 \mathrm{~g} / \mathrm{cm}^{2} \mathrm{Al}$ pressure shell

\begin{tabular}{llll}
\hline Transfer & 0.901 & 1.031 & 0.521 \\
Surface Stay & 0.149 & 0.011 & 0.202 \\
Total & $\mathbf{1 . 0 5}$ & $\mathbf{1 . 0 4 2}$ & $\mathbf{0 . 7 2 3}$ \\
Career limit: & & & \\
female \% & 117 & 116 & 80 \\
male \% & 70 & 69 & 48 \\
Annual & & & \\
limit \% & 210 & 208 & 145 \\
\hline
\end{tabular}

Setup 2: S/C with subseq. $10 \mathrm{~g} / \mathrm{cm}^{2}$ PE

\begin{tabular}{llll}
\hline Transfer & 0.632 & 0.704 & 0.335 \\
Surface Stay & 0.149 & 0.011 & 0.202 \\
Total & $\mathbf{0 . 7 8 1}$ & $\mathbf{0 . 7 1 5}$ & $\mathbf{0 . 5 5 7}$ \\
$\begin{array}{l}\text { Career limit: } \\
\text { female \% }\end{array}$ & 87 & 79 & 62 \\
$\begin{array}{l}\text { male \% } \\
\text { Annual }\end{array}$ & 52 & 48 & 37 \\
limit \% & 156 & 143 & 111 \\
\hline \hline
\end{tabular}

\begin{tabular}{|c|c|c|c|}
\hline \multicolumn{4}{|c|}{ Total mission dose eq. $[\mathrm{Sv}]$ to BFO (Solar max) } \\
\hline \multicolumn{4}{|c|}{ Setup 1: S/C with $1 \mathrm{~g} / \mathrm{cm}^{2}$ Al pressure shell } \\
\hline Transfer & 0.344 & 0.384 & 0.194 \\
\hline Surface Stay & 0.077 & 0.005 & 0.103 \\
\hline SPE & 1.80 & 1.8 & 1.8 \\
\hline SPE (shelter) & 0.243 & 0.243 & 0.243 \\
\hline Total & 2.22 & 2.189 & 2.097 \\
\hline $\begin{array}{l}\text { Total } \\
\text { (shelter) }\end{array}$ & 0.664 & 0.632 & 0.54 \\
\hline Career limit: & & & \\
\hline $\begin{array}{l}\text { female } \% \\
\text { male } \%\end{array}$ & $\begin{array}{l}74(247) \\
44(148)\end{array}$ & $42(146)$ & $\begin{array}{l}\text { bu }(233) \\
36(140)\end{array}$ \\
\hline Annual & & & \\
\hline limit \% & $133(445)$ & $126(438)$ & $108(419)$ \\
\hline \multicolumn{4}{|c|}{ Setup 2: S/C with subseq. $10 \mathrm{~g} / \mathrm{cm}^{2} \mathrm{PE}$} \\
\hline Transfer & 0.252 & 0.281 & 0.142 \\
\hline Surface Stay & 0.077 & 0.005 & 0.103 \\
\hline SPE & 0.155 & 0.115 & 0.155 \\
\hline Total & 0.484 & 0.441 & 0.4 \\
\hline Career limit: & & & \\
\hline female $\%$ & 54 & 49 & 44 \\
\hline male $\%$ & 32 & 29 & 27 \\
\hline Annual & & & \\
\hline limit \% & 97 & 88 & 80 \\
\hline
\end{tabular}

Table 11: Total mission dose equivalent $\mathrm{H}$ comparison [Sv] for selected mission scenarios and shield setups and relation to NCRP limits 


\subsection{Shield Design Proposals \& Effectiveness}

A variety of different structural design configurations has been examined to improve the protection and minimize the shield mass by the same time. When using alternative materials, a careful consideration on the various design requirements concerning structural and thermal integrity, cabin environmental criteria such as air quality, flammability and toxicity, fabrication, assembly and costs needs to be set. These issues will be taken into account briefly, while they are not considered as a main focus within this study.

The basic shield designs analyzed in terms of their overall performance are as follows:

- Basic Al structure: The qualitative dose assessment after Al2024 alloy has shown that material thicknesses currently used for pressure shells in LEO even increase high quality radiation. Although the dose received is further reduced within a fully equipped $\mathrm{S} / \mathrm{C}, \mathrm{Al}$ can not be favoured.

- Basic Al structure with PE added to the internal walls:

This setup promises good performance against ionizing radiation fields. The secondaries produced in the primary $\mathrm{Al}$ layer (mostly neutrons and protons) will be attenuated in the subsequent PE bricks. However, the PE has a parasitic shield character as long as it only fulfills radiation protective purposes and has no contribution to the structural stability.

- Combined Al-PE structure in a multi layer sandwich construction:

This design could outreach the common orthotropic stiffened aluminum shell due to a more homogenious distribution of matter over the primary walls. The sandwich could be fabricated of two outer $\mathrm{Al}$ liners and inner $\mathrm{PE}$ pad. The PE thickness can be increased to meet shield requirements without losing the typical improved sandwich stability.

- Basic Al structure with external watertanks: Water has shown to provide medium performance in radiation shielding. Outer water tanks are obligatory for a long-term manned mission, even though the water may be reconditioned in an internal cycle. Impacts of a significant mass of watertanks on the structural stability must be considered. The Al backwall could enhance production of secondary particles.

- Basic Al structure with internal watertanks: Deploying watertanks inside the $\mathrm{Al}$ pressure shell is preferable against the latter one, because the subsequent water attenuates the secondary radiation established in the Al layer. Due to lack of space inside the primary shell, watertanks could be applied at least around the crew quarters for adequate shielding during rest periods.

\section{Conclusions}

Within this engineering design study, a baseline configuration of a cylindrical spacecaft $(6 \mathrm{~m}$ diameter) for interplanetary transfer has been established. This setup was utilized to determine the attenuation of the cosmic radiation environment through the materials of the basic structure, without any additional internal or external equipment, supplies or technical devices. This first approach provides a qualitative estimation of the particle modulation within the spacecraft and the applied doses for the chosen specific materials. The results showed, that raising shield thickness of $\mathrm{Al}$ is impractical because of the increased production of secondary neutrons within the shield, which contributes significantly to the exposure. Desirable shielding materials must possess a predominant hydrogen content to minimize the production of secondary particles. According to the dependancy of the GCR particle flux on solar activity, the Mission time-frame during the solar cycle showed a significant impact on mission planning. Deploying an additional storm shelter to the S/C design can significantly reduce the dose received by the astronauts during a solar particle event (about 85\%). This reduction can only be guaranteed when an appropriate warning-time from mission control on earth or autonomous onboard sensors is assumed. Current solar observation of sun flare activity from earth-bound stations and orbital satellites allow lead times of about a few hours [27, but travelling 
time of radio signals to the crew must be allowed for. Special emphasis must be set on the placement of the shelter inside the primary structure, surrounding the crew quarters with $10 \mathrm{~g} / \mathrm{cm}^{2} \mathrm{PE}$ or drinking water is beneficial, although the latter will be consumed during transfer time. Polyethylenefiber reinforced composites show great potential to meet both safety from ionizing radiation and good structural integrity to be applied as a ballistic shield.

It was shown, that independently from the exact type or chemical composition of the shielding material, any passive shielding solution will require a certain amount of areal density to reduce the expected crew exposures to acceptable levels.

\section{Discussion}

Most of the analyzed materials have the character of a parasitic shield ( $\mathrm{LiH}$ and the polymers PE, PSO and PEI), which means they have no further function within the design than shielding from radiation and thus give additional weight to the configuration. Nevertheless, PSO and PEI are included in the analysis, although they show less attenuation against radiation than polyethylene, but possess a better structural integrity. This gives them a certain significance within the design process. $\mathrm{LiH}$ is one of the best hydrogen storage materials currently available, however it has problematic features as it is easy flammable, toxic and highly reactive to water. HGNF has not been fully established as an applicable material and has an exploratory status, but it was included into this study to give a future perspective. CFRP is used within recent aircraft designs more frequently and establishes as an alternative to aluminum. The usage of polyethylene-fiber reinforced composites promises to combine excellent radiation shield performance with the required mechanical properties of structural materials.

For Mars surface stay the use of martian regolith could be an interesting alternative, while the use of polyimides and polyethylene as binders of regolith for developement of basic structural elements would even enhance their protective properties [18].

In the course of this study, certain limitations appeared in terms of establishing a detailed dose assessment for the depicted mission scenarios. This originated partly from ongoing elaboration of essential radiation transport codes and affiliated programs for precise valuation of cosmic radiation spectra, simulation of planetary atmospheric models and provision of appropriate anatomical human targets. Additionally, implementation of the effective dose equivalent $\mathrm{E}$ and estimation of health risks are widely uncertain due to the limitations in radiobiological data and knowledge, especially for HZE ions. With regard to long-term damaging effects, the reaction of the human body on various kinds of radiation must be decoupled from other harmful implications during lifetime. Thus estimating the expected risks from radiation fields in deep space and on the Mars surface relies on further understanding of the biological response within human cells and tissue.

Future work will focus on these issues, calculations for effective dose will be performed using detailed CAD geometries and thickness definitions of the $\mathrm{S} / \mathrm{C}$ and habitat structure as well as reliable spectra for the radiation environment on Mars. Shield performance of further multilayered material setups like sandwich structures will be investigated. The additional placement of equipment in and outward the basic structure in order to optimize astronaut's shielding and minimize parasitic shield requirements will be investigated in a future detailed design study.

\section{Acknowledgement}

The author would like to thank Dr. Robert C. Singleterry, Administrator's Fellow at NASA Langley Research Center, for his invaluable support and provision of results from the HZETRN code, Dr. Hugh Evans from the ESA/ESTEC Space Environment and Effects Section for his patience and endless discussions, Dr. Fan Ley from Qinetiq for precious support in utilizing Geant4/Mulassis and Dipl.-Ing. Michael Quatmann and Dipl.-Ing. Daniel Noelke from the Department of Aerospace and Lightweight Structures of the RWTH-Aachen University for paving the way to apply this study.

For further detailed information on the addressed field of research and our related studies please contact Mr. Andreas Borggraefe (E-Mail address: Andreas.Borggraefe@rwth-aachen.de). 


\section{References}

[1] NASA, Human Exploration of Mars: the Reference Mission of the NASA Mars Exploration Study Team, NASA SP-6107, 1997.

[2] J.W. Wilson, F.F. Badavi, F.A. Cucinotta, Standardized radiation shield design method: 2005 HZETRN, SAE International, 2006.

[3] F. Lei, P.R. Truscott, C.S. Dyer, B. Quaghebeur, D. Heynderickx, P. Nieminen, H. Evans, E. Daly, MULASSIS: A Geant4-based multilayered shielding simulation tool, IEEE Transactions on Nuclear Science, Vol. 49, No. 6, 2002.

[4] National Research Council: Space Radiation Hazards and the Vision for Space Exploration: Report of a Workshop (2006).

[5] J.W. Wilson, J. Miller, A. Konradi, F.A. Cucinotta, Shielding strategies for human space exploration, NASA Conference Publication 3360, 1997.

[6] J.W. Wilson, F.A. Cucinotta, J.L. Shinn, L.C. Simonsen, R.R. Dubbed, W.R. Jordan, T.D. Jones, C.K. Chang, M.Y. Kim, Shielding from solar particle event exposures in deep space, Radiation Measurements 30, pp. 361-382, 1999.

[7] W. Stolz, Radioaktivität, 5. Edition, Teubner Publishing, 2005.

[8] E.R. Benton, E.V. Benton, Space Radiation dosimetry in low-Earth orbit and beyond, Nuclear Instruments and Methods in Physics Research B 184, pp. 255-294, 2001.

[9] T. Berger, Dose assessment in mixed radiation fields, Special emphasis on space dosimetry, PhD, Vienna, Austria, 2003.

[10] ICRP, Recommendations of the international commission on radiological protection 26, Pergamon Press, Oxford, 1977.

[11] ICRP, Recommendations of the international commission on radiological protection 60, Pergamon Press, Oxford, 1991.

[12] L.W. Townsend, R.J.M. Fry, Radiation protection guidance for activities in low-earth orbit, Advances in Space Research, Vol. 30, No. 4, 2002.

[13] National Council on Radiation Protection and Measurement, Guidance on radiation received in space activities, NCRP Report No. 98, Bethesda, MD, 1989.

[14] National Council on Radiation Protection and Measurement, Radiation protection guidance for activities in low-earth orbit, NCRP Report No. 132, Bethesda, MD, 2000 .

[15] J.L. Shinn, F.A. Cucinotta, R.C. Singleterry, J.W. Wilson, F.F. Badavi, G.D. Badhwar, J. Miller, C. Zeitlin, L. Heilbronn, R.K. Tripathi, M.S. Clowdsley, J.H. Heinbrockel, A radiation shielding code for spacecraft and its validation, Johnson Space Center, Langley Research Center, NASA, 2000.
[16] J.L. Shinn, F.A. Cucinotta, R.C. Singleterry, J.W. Wilson, F.F. Badavi, G.D. Badhwar, J. Miller, C. Zeitlin, L. Heilbronn, R.K. Tripathi, M.S. Clowdsley, J.H. Heinbrockel,M. A. Xapsos, Validation of a Comprehensive Space Radiation Transport Code, IEEE Transactions On Nuclear Science, Vol. 45, No. 6, 1998.

[17] M.P. Billings, W.R. Yucker, The computerized anatomical man (CAM) model. NASA CR-134043, 1973.

[18] J.W. Wilson, F.A. Cucinotta, R.K. Tripathi, et. al., Improved Spacecraft Materials for Radiation Protection,Johnson Space Center, Langley Research Center, USA, 2001.

[19] A.F. Barghouty, R.K. Kaul, H.M. Dahche, Space radiation transport properties of polyethylene-based composites, Annals of the New York Academy of Sciences 1027 (1), pp. 138-149, 2004.

[20] A.F. Barghouty, S.A. Thibeault, The exploration atmospheres working group's report on space radiation shielding materials, Langley Research Center, Marshall Space Flight Center, NTRS, 2006.

[21] P.M. O'Neill, Badhwar-O'Neill galactic cosmic ray model update based on advanced composition explorer (ACE) energy specra from 1997 to present, Johnson Space Center, NASA, 2005.

[22] J.H. King, Solar proton fluences for 1977-1983 space missions, Journal of Spacecraft and Rockets, vol. 11, pp. 401-432, 1974.

[23] J.W. Wilson, F.A. Cucinotta, H. Tai, L.C. Simonsen, J.L. Shinn, S.A. Thibeault, M.Y. Kim, Galactic and solar cosmic ray shielding in deep space, NASA Technical Paper 3682, 1997.

[24] L.C. Simonsen, Analysis of lunar and mars habitation modules for the space exploration initiative (SEI), in 'Shielding Strategies for Human Space Exploration', Edited by J.W. Wilson, J. Miller, A. Konradi, and F. A. Cucinotta, NASA Conference Publication 3360, 1997.

[25] P.B. Saganti, F.A. Cucinotta, J.W. Wilson, L.C. Simonsen,C. Zeitlin, Radiation climate map for analyzing risks to astronauts on the mars surface from galactic cosmic rays, Space Science Reviews, Volume 110, 2002.

[26] D. Rapp, Radiation effects and shiedling rquirements in human missions to the moon and mars, The International Journal of Mars Science and Exploration, Mars 2, pp.46-71, 2006.

[27] J. Feynman, A. Ruzmaikin, Problems in the forecasting of solar particle events for manned missions, Radiation Measurements 30, pp. 275-280, 1999. 